\title{
Engineering Calculation of Double Closed-loop Controller Parameters of Chopper Cascade Speed System
}

\author{
Shiyang Su \\ School of North China Electric Power University,Baoding 071003 Hebei Provice China; \\ Sushiyang88@sina.com
} Keywords: state-space averaging method, third-order engineering best method, double
closed-loop control,collapsed material

\begin{abstract}
According to main circuit characteristics of chopper cascade control system under steady state, and system speed characteristics, mechanical characteristics, differential equations of main circuit of chopper cascade control system is established under state-space averaging method. Using the second-order and third-order engineering best method combined with practical engineering, engineering calculations method of double closed-loop controller parameters of chopper cascade speed system is detailed derived. Double closed-loop controller of chopper cascade speed system is designed under this engineering calculation approach, and is used in high-temperature fan of rotary kiln cement production line. Compared to the traditional cascade speed system, the DC bus current is better controlled. The reliability and dynamic performance of chopper cascade speed system is improved, and "collapse material" leading to trip outage of high-temperature fan, production interruptions and other issues is effectively solved.
\end{abstract}

\section{Introduction}

Considering different production process on site, some equipments need high requirements in the dynamic nature of system. For instance, zero static difference of rotate speed should be achieved under prerequisite of ensuring system stability. For another instance, the dynamic rotate speed can be reduced under load mutation. It cannot be achieved by merely using open-loop control. For single closed-loop control, it cannot meet the demands because it is hard to effectively control current and torque which leads time-consuming and over-adjustment. Double closed-loop control of rotate speed and current is an effective way in solving system dynamic and steady characteristics. This paper aims to establish differential equation in state-average model based on major loop feature under steady circumstance of chopper cascade speed control system and governing and mechanical characteristics. Furthermore, on the basis of this, it also provides the design method of double closed-loop controller parameters of chopper cascade speed system. It is widely applied on the spot, especially in high temperature fan of cement plant. The application prove that double closed-loop control can increase anti-jamming capability and safety of system , effectively restrain the bus current, prevent frequently over-current protection and improve system dynamic characteristics.

\section{Structure and Working Principle of Chopper Cascade Speed System}

The typical structure of chopper cascade speed system as shown in Figure 1.In this system, three-phase winding rotor of left-side joins into three-phase uncontrolled rectifier which consists of diodes. DC link in the middle is boost chopping circuit. The right side is active inverter that is made of inverter transformer and thyristor. This structure forms additional electromotive force and achieves feedback of slip power. This system uses three-phase winding asynchronous motor. Electric reactor L1 and L2 play smoothing effect in the loop. For Capacitor C, it can filter waves and store energy. When IGBT is in working condition under PWM signal modulation, it can be turned on or off based on duty ratio in a certain period. Additional E.M.F is short connected when 
switching on. On the contrary, additional E.M.F accesses into the main loop. The average back additional E.M.F can be manipulated by altering duty ratio in a certain period, which can lead the transformation of rotor current, change electromagnetic torque and achieve the goal of speed control.

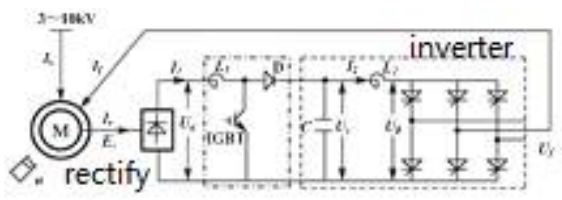

Fig.1 Chopper cascade speed system structure

\section{Dynamic Mathematical Modeling of Main Loop of Chopper Cascade Speed System}

As Figure 1 shows that main circuit of the system is typical boost chopper. The input of this is rotor rectifier voltage and inverter voltage with active inverse. The output is two inductance currents and capacitor voltage. Meanwhile, the output depends on input, structure parameter and given duty ratio. Due to high frequency of chopper and filtering waves of inductor and capacitor on higher harmonic, the average value of rotor rectifier and converter in a certain cycle is used when analyzing this circuit. When chopper cascade speed control system is under normal operation, the bus current is continuous. This paper is based on this pre-condition. According to modeling theory of nonlinear converter, the main loop of chopper cascade speed control system can be shown as two linear none-time modification of electric circuit that correspond to two turn on and off state in the cycle as shown in Figure 2. In order to conduct analysis, IGBT chopper, inner resistance(r)of diode with reverse block and forward voltage-drop $(\Delta \mathrm{u})$ should be neglected.

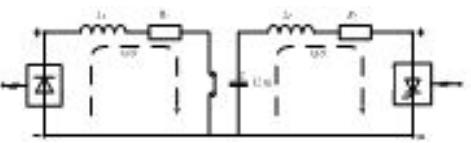

(a) Main circuit topology when IGBT turns on

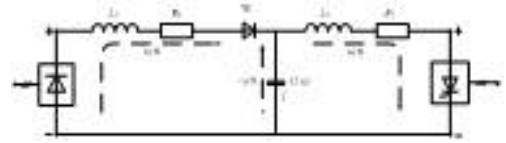

(b) Main circuit topology when IGBT turns off

Fig.2 Main circuit topology when IGBT different states

(1) When IGBT turns on, it is the first topology. Equivalent circuit as shown in Figure 2(a). In the $\mathrm{k}^{\text {th }}$ period, that is $t_{k} \leq t \leq t_{k}+\mathrm{d} T$, $\mathrm{d}$ is duty ratio of chopper, $\mathrm{T}$ is cycle. Following results can be established.

$$
\left\{\begin{array}{l}
L_{1} \frac{d i_{1}}{d t}=-i_{1} R_{1}+U_{d}(t) \\
L_{2} \frac{d i_{2}}{d t}=-i_{2} R_{2}+U_{c}(t)-U_{\beta}(t) \\
C \frac{d U_{c}(t)}{d t}=-i_{2}
\end{array}\right.
$$

In the equation, $U_{d}(t)$ is three-phase uncontrolled rectifier that can convert rotor three-phase induction electromotive force into six-pulse E.M.F based on slip ratio.

$$
U_{d}(t)=2.34 s E_{r 0}\left(1-\sum_{n=6 m}^{\infty} \frac{2 \cos m \pi}{n^{2}-1} \cos n s \omega_{1} t\right), m=1,2,3, \ldots
$$

Except fundamental wave, coefficient of harmonic component is too small that can be ignored. So, $U_{d}(t)=U_{d 0}=2.34 s E_{r 0}$

$U_{d}(t)$ represents rotor voltage open circuit potential. S is motor slip ratio.

For inverter side just like rectifier, due to minor sub-harmonic and suppression effect for ultra-harmonics, only fundamental wave is considered, other should be neglected.

Differential equations of (1) can be transformed into matrix form as (5) shown: 


$$
\left[\begin{array}{c}
\frac{d i_{1}(t)}{d t} \\
\frac{d i_{2}(t)}{d t} \\
\frac{d U_{c}(t)}{d t}
\end{array}\right]=A_{1}\left[\begin{array}{l}
i_{1}(t) \\
i_{2}(t) \\
U_{c}(t)
\end{array}\right]+B_{1}\left[\begin{array}{l}
U_{d}(t) \\
U_{\beta}(t)
\end{array}\right]
$$

i1 is the current through L1 inductor, and i1 is the current through L1 inductor. Uc is the voltage between capacitor C. Coefficient matrix of A1 and B1 is shown as:

$$
A_{1}=\left[\begin{array}{ccc}
-\frac{R_{1}}{L_{1}} & 0 & 0 \\
0 & -\frac{R_{2}}{L_{2}} & \frac{1}{L_{2}} \\
0 & -\frac{1}{C} & 0
\end{array}\right], B_{1}=\left[\begin{array}{cc}
\frac{1}{L_{1}} & 0 \\
0 & -\frac{1}{L_{2}} \\
0 & 0
\end{array}\right] 。
$$

(2) When IGBT turns off, it is the second topology. Equivalent circuit as shown in Figure 2(b). In the $\mathrm{k}^{\text {th }}$ period, that is $t_{k}+\mathrm{d} T \leq t \leq t_{k+1}$. Following results can be established.

$$
\left\{\begin{array}{l}
L_{1} \frac{d i_{1}}{d t}=-i_{1} R_{1}-U_{c}(t)+U_{d}(t) \\
L_{2} \frac{d i_{2}}{d t}=-i_{2} R_{2}+U_{c}(t)-U_{\beta}(t) \\
C \frac{d U_{c}(t)}{d t}=i_{1}-i_{2}
\end{array}\right.
$$

Simplify the differential equations in the above-mentioned way, the following matrix form can be made.

$A_{2}=\left[\begin{array}{ccc}-\frac{R_{1}}{L_{1}} & 0 & -\frac{1}{L_{1}} \\ 0 & -\frac{R_{2}}{L_{2}} & \frac{1}{L_{2}} \\ \frac{1}{C} & -\frac{1}{C} & 0\end{array}\right], B_{2}=\left[\begin{array}{cc}\frac{1}{L_{1}} & 0 \\ 0 & -\frac{1}{L_{2}} \\ 0 & 0\end{array}\right]$ 。

$$
\left[\begin{array}{l}
\frac{d i_{1}(t)}{d t} \\
\frac{d i_{2}(t)}{d t} \\
\frac{d U_{c}(t)}{d t}
\end{array}\right]=A_{2}\left[\begin{array}{l}
i_{1}(t) \\
i_{2}(t) \\
U_{c}(t)
\end{array}\right]+B_{2}\left[\begin{array}{l}
U_{d}(t) \\
U_{\beta}(t)
\end{array}\right]
$$

From the above, (5) and (7) are state equations of the continues system. Take $X(t)=\left[\begin{array}{lll}d i_{1}(t) / d t & d i_{2}(t) / d t & d U_{c}(t) / d t\end{array}\right]^{T}$ as state variable, (5) can be made based on modern control theory of state equations of the continues system(when IGBT is on).

$$
X(t)=e^{A_{1}\left(t-t_{k}\right)} X\left(t_{k}\right)+A_{1}^{-1}\left(e^{A_{1}\left(t-t_{k}\right)}-I\right) B_{1} U
$$

$U=\left[\begin{array}{l}2.34 s E_{r 0} \\ 2.34 U_{f} \cos \beta_{\min }\end{array}\right]$ 。

When system is under the second topology(when IGBT is off), that is $t_{k}+d T \leq t \leq t_{k+1}$, (7) can be shown as:

$$
\begin{aligned}
X(t)= & e^{A_{2}\left[t-\left(t_{k}+d T\right)\right]} X\left(t_{k}+d T\right) \\
& +A_{2}^{-1}\left(e^{A_{2}\left[t-\left(t_{k}+d T\right)\right]}-I\right) B_{2} U
\end{aligned}
$$

Based on (8), the final value of the first topology can be written as:

$$
X\left(t_{k}+d T\right)=e^{A_{1} d T} X\left(t_{k}\right)+A_{1}^{-1}\left(e^{A_{1} d T}-I\right) B_{1} U
$$

Based on (9), the final value of the first topology can be written 
as: $\begin{aligned} X\left(t_{k+1}\right)= & e^{A_{2}(1-d) T} X\left(t_{k}+d T\right) \\ & +A_{2}^{-1}\left(e^{A_{2}(1-d) T}-I\right) B_{2} U\end{aligned}$

Because the variables of inductive current and capacitor voltage in the system are continuous, that is the initial value of the second topology is the final value of the first one. Therefore, substitute (9) into (11), the state variable of the kth is: $\begin{gathered}X\left(t_{k+1}\right)=e^{\left[A_{2}(1-d)+A_{d} d\right] T} X\left(t_{k}\right)+e^{A_{2}(1-d) T} A_{1}^{-1}\left(e^{A_{1} d T}-I\right) B_{1} U \\ +A_{2}^{-1}\left(e^{A_{2}(1-d) T}-I\right) B_{2} U\end{gathered}$

For high frequency chopper, the wave frequency under engineering application is $1.35 K \leq f \leq 2.7 \mathrm{~K}$. Because the period is too short, it can be replaced by $e^{A T} \cong I+A T$ that is the linear expression of exponential function. The result of termination system of switching period can be written as:

$$
\begin{aligned}
X\left(t_{k+1}\right)-X\left(t_{k}\right)= & {\left[A_{1} d+A_{2}(1-d)\right] T X\left(t_{k}\right) } \\
& +\left[d B_{1}+(1-d) B_{2}\right] T U
\end{aligned}
$$

(13) should be marked as the standard form $\dot{X}=A X+B U$. New coefficient matrix of the state equation are linear combination of two sub-system, that is, $\left\{\begin{array}{l}A=\left[d A_{1}+(1-d) A_{2}\right] \\ B=\left[d B_{1}+(1-d) B_{2}\right]\end{array}\right.$

From the equation, the contribution of whole cycle response in every state is described as $A_{1}+d T$ and $A_{2}+(1-d) T$.Therefore, state space description under average features can be made in a switching period that state and input variables are remain unchanged.

$$
\left[\begin{array}{c}
\frac{d i_{1}(\mathrm{t})}{d t} \\
\frac{d i_{2}(\mathrm{t})}{d t} \\
\frac{d U_{c}(\mathrm{t})}{d t}
\end{array}\right]=\left[\begin{array}{ccc}
-\frac{R_{1}}{L_{1}} & 0 & -\frac{1-d}{L_{1}} \\
0 & -\frac{R}{L_{2}} & \frac{1}{L_{2}} \\
\frac{1-d}{C} & -\frac{1}{C} & 0
\end{array}\right]\left[\begin{array}{c}
i_{1}(\mathrm{t}) \\
i_{2}(\mathrm{t}) \\
U_{c}(\mathrm{t})
\end{array}\right]+\left[\begin{array}{cc}
\frac{1}{L_{1}} & 0 \\
0 & -\frac{1}{L_{2}} \\
0 & 0
\end{array}\right]\left[\begin{array}{l}
U_{d}(t) \\
U_{\beta}(t)
\end{array}\right]
$$

(15) can be written as following loop in a average current and voltage under chopper sampling.

$$
\begin{gathered}
L_{1} \frac{d i_{1}(t)}{d t}+i_{1}(t) R_{1}=U_{d}(t)-(1-d) U_{c}(t) \\
L_{2} \frac{d i_{2}(t)}{d t}+i_{2}(t) R_{2}=U_{c}(t)-U_{\beta}(t) \\
C \frac{d U_{c}(t)}{d t}=(1-d) i_{1}(t)-i_{2}(t)
\end{gathered}
$$

The following equation can be deduced as: $i_{2}(t)=(1-d) i_{1}(t)-C \frac{d U_{c}(t)}{d t}$

Make derivation of (19) can be written as, $\frac{d i_{2}(t)}{d t}=(1-d) \frac{d i_{1}(t)}{d t}-C \frac{d^{2} U_{c}(t)}{d t}$

$$
\begin{aligned}
& L_{2}(1-d) \frac{d i_{1}(t)}{d t}-L_{2} C \frac{d^{2} U_{c}(t)}{d t}+R_{2}(1-d) i_{1}(t) \\
& -R_{2} C \frac{d U_{c}(t)}{d t}=U_{c}(t)-U_{\beta}(t)
\end{aligned}
$$

From (21), if L2 and C are both $10^{-3}$ grade and $R_{1}$ is $10^{-1}$ and $R_{2}$ is $10^{-2}$ in the main loop. Under this condition, $L_{2} C$ and $R_{2} C$ are too small, then (21) can be transformed into,

$$
\begin{aligned}
& L_{2}(1-d) \frac{d i_{1}(t)}{d t}+R_{2}(1-d) i_{1}(t)=U_{c}(t)-U_{\beta}(t) \\
& \left.\left[L_{1}+(1-d)^{2} L_{2}\right] \frac{d i_{1}(t)}{d t}+\left[R_{1}+(1-d)^{2} R_{2}\right]\right]_{1}(t) \\
& =U_{d}(t)-(1-d) U_{\beta}(t)
\end{aligned}
$$

$i_{1}(t)$ of DC bus current and $U_{d}(t)$ and $U_{\beta}(t)$ of input voltage can be seen in average dynamic relation under sampling period in (23).

$\begin{array}{cccc}\text { Make Laplace transform } & \text { for } \quad(23), & \text { because } & \mathrm{Ud}(\mathrm{t})=\mathrm{Ud} 0 \text {, } \\ \mathrm{U} \beta(\mathrm{t})=\mathrm{U} \beta 0, \frac{U_{d 0}-(1-d) U_{\beta 0}}{\left[L_{1}+(1-d)^{2} L_{2}\right] s+\left[R_{1}+(1-d)^{2} R_{2}\right]}=I_{1}(s) & \text { (24) }\end{array}$


If RVT of IGBT chopper equals RVD of reverse blocking diode, that is RVT $=\mathrm{RVD}=\mathrm{RV}$, with which has been converted into RD of asynchronous motor on the rotor side, inverter transformer of the secondary side or equivalent resistance RT of feedback motor winding side and equivalent resistance $3 s X_{D} / \pi$ and $3 X_{T} / \pi$ of rotor and inverter sides. All of these have been included into R1 and R2. Then use RL1 and RL2 instead of R1 and R2, that is $R_{L 1}=R_{1}+R_{V}+\frac{3 s X_{D}}{\pi}+1.7 R_{D} \quad R_{L 2}=\left(R_{2}+\frac{3 X_{T}}{\pi}+1.7 R_{T}\right)$.Then let DC component $2.34 s E_{r 0}$ of $U_{d}(t)$ and $2.34 U_{f} \cos \beta_{\min }$ of $U_{\beta}(t)$ into (23) finding that $\quad L_{\Sigma} \cdot \frac{d i_{1}(t)}{d t}+R_{s \Sigma} \cdot \dot{i}_{1}(t)=2.34 \cdot\left[s E_{r 0}-(1-d) U_{f} \cdot \cos \beta_{\min }\right]$

式中, $R_{s \Sigma}=R_{L 1}+(1-d)^{2} R_{L 2} ; \quad L_{\Sigma}=1.7 L_{D}+L_{1}+(1-d)^{2} \cdot\left(L_{2}+1.7 L_{T}\right) ; \quad L_{D}=X_{D} / 2 \pi f_{1}, L_{T}=X_{T} / 2 \pi f_{1}$ 。

Take $s=\left(n_{1}-n\right) / n_{1}$ into (25), then use Laplace Transform. Transfer Function of chopper cascade speed control in the main loop has been made. $G(s)=\frac{I_{1}(s)}{2.34 \cdot\left[E_{r 0}\left(1-n(s) / n_{1}\right)-(1-d) U_{f} \cos \beta_{\min }\right]}=\frac{K_{L n}}{T_{L n} s+1}$

$T_{L n}$ in this equation is time constant of direct loop $T_{L n}=L_{\Sigma} /\left(R_{\Sigma}-3 X_{D} n / \pi n_{1}\right) . K_{L n}$ is amplification system $K_{L n}=1 /\left(R_{\Sigma}-3 X_{D} n / \pi n_{1}\right) \quad$. $R_{\Sigma} \quad$ is equivalent resistance of the main loop $R_{\mathrm{E}}=3 X_{\mathrm{D}} / \pi+1.7 R_{\mathrm{D}}+R_{\mathrm{V}}+R_{1}+(1-d)^{2} \cdot\left(3 X_{\mathrm{T}} / R_{\mathrm{T}}+1.7 R_{\mathrm{T}}+R_{2}\right) \quad$ when $s=1$. And $n_{1}$ is synchronous speed.

Till then, dynamic relation of duty ratio of the main loop in the chopper cascade, structure parameter and load DC in an average switching period has been deduced. Because rotor current is related to load DC, then determine torque and speed.

Electromagnetic torque of the chopper cascade system is $T_{e}=\frac{1}{\omega_{1}}\left(2.34 E_{20}-\frac{3 X_{D}}{\pi} I_{1}\right) \cdot I_{1}$

$\omega_{1}$ is synchronous speed. Because torque expression is non linear equation, Taylor expansion has been used in the steady site(determine by given speed $n^{*}$ and load torque $T_{L}$ ). Considering that second derivative

$d^{2} T_{e} / d I_{1}^{2}=-\left(1 / \omega_{1}\right)\left(6 X_{D} / \pi\right)$ is too small which can be neglected, only extend the first. $T_{e}=T_{0}+\frac{1}{\omega_{1}}\left(2.34 E_{r 0}-\frac{6 X_{D}}{\pi} I_{10}\right)\left(I_{1}-I_{10}\right)$

$T_{0}$ is electromagnetic torque in the steady working site. $I_{10}$ is average DC. If $C_{0}=\frac{1}{\omega_{1}}\left(2.34 E_{20}-\frac{6 X_{D}}{\pi} I_{d 0}\right)$ at the same time let equation, that is

$C_{0}\left(I_{1}-I_{1 L}\right)=\frac{G D^{2}}{375} \frac{d n}{d t}$

$I_{1 L}$ is DC of load torque. Then use Laplace transform of the both sides, that is $\frac{n(s)}{I_{1}(s)-I_{1 L}(s)}=\frac{1}{T_{I} s}$ (30)

For $T_{I}=G D^{2} / 375 C_{0}, G D^{2}$ is flywheel inertia for the motion part.

Due to the delay from PWM impulse to switch action, there is a carrier wave lag from duty ratio converting into DC bus. For the convenience, that can be seen as one order inertial link, time constant is Ts, magnification factor is $\mathrm{Kv}$, that is $\quad G_{f}(s)=\frac{K_{v}}{T_{s} s+1}$

Draw the transfer function schema of (26),(30) and (31). Considering given filter wave and feedback filter wave links, the dynamic schema of double closed-loop cascade speed control system can be made.

\section{Calculation of Double Closed-loop Controller Parameters}

4.1 Calculation of Current Regulator Parameters

Current detection mainly applies DC transformer or hall effect transducer. In this paper, the 
detection method is that using hall effect transducer measures impulse current of DC bus. Then use number filter wave of average sampling in the second chopper period. Filtering time constant is always between 1 to 2 millisecond.

The respond speed of current is more faster than rotor speed. The change of rotor speed is very tiny in the process of current adjustment process. Therefore, electromotive force $2.34 E_{r 0} \cdot n(s) / n_{1}$ can be neglected, that is circuit branch switching off. Then, let feedback link move into inner loop. The unit negative feedback is made.

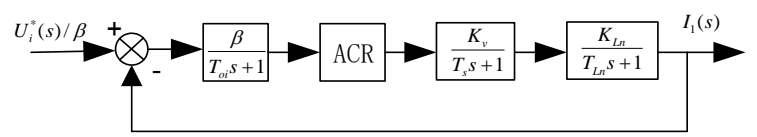

Fig.4 Simplified diagram of the current loop dynamic structure with the type I

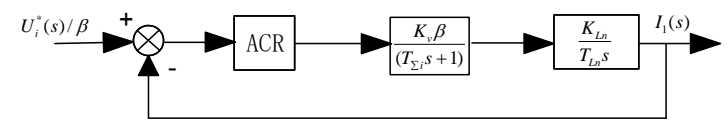

Fig.5 Simplified diagram of the current loop dynamic structure with the type II

Due to the delay of wave carrier cycle from PWM impulse to DC bus, current feedback time constant is small that can be seen as small inertia time constant, that is $T_{\Sigma i}=T_{s}+T_{o i}$.

When conducting current correction, following behaviors should be considered. The less overshoot, the better. So, type 1 system design current loop should be applied. For example,

(1) Conduct the design of type 1 system, transfer function of current loop controller is

$W_{A C R}(s)=K_{i} \cdot \frac{\tau_{i} s+1}{\tau_{i} s}$

In order to eliminate the correspond pole of inertia time constant, if $\tau_{i}=T_{L n}$, the type I of expected transfer function of open loop can be deduced. $\frac{K_{i} K_{V} K_{L n} \beta}{\tau_{i} S\left(T_{\Sigma i} s+1\right)}=\frac{K}{s(T s+1)}$

According to (33), $\quad T=T_{\Sigma i}$ and $K=K_{i} K_{V} K_{L n} \beta / \tau_{i}$ can be established. And $\tau_{i}=T_{L n}$, so $\quad K=K_{i} K_{V} K_{L n} \beta / T_{L n} . \quad \beta$ is current reaction coefficient.

Generally speaking, engineering design expects that current overshoot is $\sigma_{i} \leq 5 \%$. At the same time, $\quad \xi=0.707, K T=0.5$, the controller parameter of current loop PI is, $\left\{\begin{array}{l}K_{i}=\frac{T_{L n}}{2 K_{V} K_{L n} \beta T_{\Sigma i}} \\ \tau_{i}=T_{L n}\end{array}\right.$

Conduct the design of type II. Big inertia time constant can approximately be seen as integration element(on the condition that $T_{L n}$ of big inertial element is much larger than $T_{\Sigma i}$ ). Therefore, $K_{L n} /\left(T_{L n} s+1\right) \approx K_{L n} / T_{L n} s \quad$ combining with small inertia time constant, the expected transfer function of type II in the open loop can be made: $K_{i} \frac{\tau_{i} s+1}{\tau_{i} s} \times \frac{K_{V} K_{L n} \beta}{T_{L n} s\left(T_{\Sigma i} s+1\right)}=\frac{K(\tau s+1)}{s^{2}(T s+1)}$

From (35) can be deduced that $\left\{\begin{array}{l}K=\frac{K_{i} K_{V} \beta K_{L n}}{\tau_{i} T_{L n}} \\ T=T_{\Sigma i} \\ \tau=\tau_{i}\end{array}\right.$

Based on the requirements of tracing performance and disturbance restraint performance, IF Wideband is $h=5$, the followings can be made: $\left\{\begin{array}{l}K=\frac{(h+1)}{2 h^{2} T^{2}}=\frac{3}{25 T^{2}} \\ \tau=h T=5 T\end{array}\right.$

Substitute (36) into (37). Controller parameters of PI can be deduced according to type II optimum engineering design. $\left\{\begin{array}{l}K_{i}=\frac{3 T_{L n}}{5 K_{V} K_{L n} T_{\Sigma i} \beta} \\ \tau_{i}=5 T_{\Sigma i}\end{array}\right.$

From open-loop transfer constant $\frac{K(\tau s+1)}{s^{2}(T s+1)}$ of type II, the closed-loop transfer function can be 
written as

$$
G(s)=\frac{K(\tau s+1)}{T s^{3}+s^{2}+K \tau s+K}
$$

The closed-loop transfer function of type II exceeds one zero, that is $\tau s+1$. Therefore, time constant is should add one zero. Null point can offset to avoid overshoot. $W_{g i}(s)=\frac{1}{T_{g i} s+1}=\frac{1}{\left(5 \times T_{\Sigma i}+T_{o i}\right) s+1}$

In conclusion, current loop is based on optimal second order engineering or optimum third design dynamic parameter, which low frequency great inertial link can approximately treat as integration. Chopper cascade control system can be applied as follows,

(1) When $T_{L n} \leq 4 T_{\Sigma i}$, current dynamic parameter is designed according to second order engineering optimum(type 1 system structure).

(2) When $4 T_{\Sigma i}<T_{L n}<30 T_{\Sigma i}$, current dynamic parameter is designed according to second order engineering optimum . The larger $T_{L n} / T_{\Sigma i}$, the rectified performance index is close to performance index of third engineering optimum; on the contrary, the rectified performance index is close to performance index of second engineering optimum.

4.2 Calculation of Speed Regulator

For speed control system, the most important dynamic property is anti-confuse capacity, such as anti-load disturbance and anti-network and voltage fluctuation. But from the perspective of dynamic performance, if load disturbance action point is after current loop, can only use speed regulator ASR to restrain. When designing ASR, anti-interference performance should be used. Meanwhile, static performance index meets floating control. Therefore, closed-loop speed rotor uses type II system. The structure of closed-loop controller of chopper cascade speed system and reduced form are shown in Figure 6.

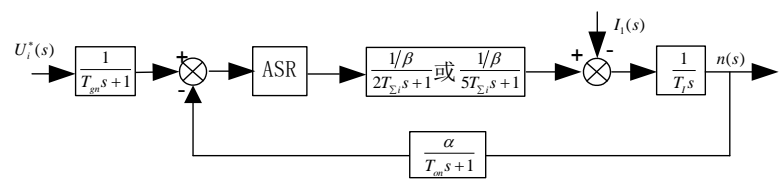

Fig.6 Simplified diagram of chopper cascade speed system speed loop dynamic structure (1) Equivalent transfer function of current loop is based on type I system design.

$$
W_{i c l}(s)=\frac{\frac{K}{s\left(T_{\Sigma i} s+1\right)}}{1+\frac{K}{s\left(T_{\Sigma i} s+1\right)}}=\frac{1}{\frac{T_{\Sigma i}}{K} s^{2}+\frac{s}{K}+1}
$$

Because $T_{\Sigma i} / K$ is so small, reduced order is made for (41). Then current closed-loop transfer constant is made, that is $W_{i c l}(s)=\frac{1 / \beta}{2 T_{\Sigma i} s+1}$

(2)Equivalent transfer function of current loop is based on current loop. Substitute (36) into (39), then closed-loop transfer constant is made, $W_{i c l}(s)=\frac{5 T_{\Sigma i} s+1}{8.33 T_{\Sigma i}^{3} s^{3}+8.33 T_{\Sigma i}^{2} i^{2}+5 T_{\Sigma i} s+1}$

Equivalent transfer function after using inertial element of current filter wave and offset

$$
\begin{aligned}
& \text { differentiation element of (43) can be simplified as } W_{i c l}(s)=\frac{1 / \beta}{5 T_{\Sigma i} s+1} \times \frac{5 T_{\Sigma i} s+1}{8.33 T_{\Sigma i}^{3} s^{3}+8.33 T_{\Sigma i}^{2} s^{2}+5 T_{\Sigma i} s+1} \\
& \approx \frac{1 / \beta}{5 T_{\Sigma i} s+1}
\end{aligned}
$$

Current loop is inner ring and revolving speed loop is outer ring. Substitute (42) or (44) as inertial element into speed loop. If transfer function of speed loop is $W_{A S R}(s)=K_{n} \frac{\tau_{n} s+1}{\tau_{n} s}$

Referring to Figure 6 and make adjustment of inertial element and bring the feedback into forward path, that is $T_{\Sigma n}=2 T_{\Sigma i}+T_{o n}$ 
The simplified open-loop transfer constant of type II is $\quad K_{n} \frac{\tau_{n}+1}{\tau_{n} s} \times \frac{\alpha /\left(\beta T_{I}\right)}{s\left(T_{\Sigma n} s+1\right)}=\frac{K(\tau s+1)}{s^{2}(T s+1)}$

According to engineering optimal design method of type II, the condition of $\tau$ and $K$ in (37) can deduce the controller parameter of PI in rotate speed loop. $\left\{\begin{array}{l}K_{n}=\frac{(5+1) \beta T_{I}}{2 \times 5 a T_{\Sigma n}}=\frac{0.6 \beta T_{I}}{a T_{\Sigma n}} \\ \tau_{n}=\tau=5 T_{\Sigma n}\end{array}\right.$

Similarly with the above-mentioned analysis, closed-loop transfer function of type II will add a zero $\tau s+1$. In order to offset, add a time constant $\tau s$ as inertial element in the revolving speed loop. Then transfer function of the given filter wave in the outer loop is $W_{g n}(s)=\frac{1}{T_{g n} s+1}=\frac{1}{\left(5 \times T_{\Sigma n}+T_{o n}\right) s+1}$

\section{References}

[1] SHANG Debin, LIU Huawei,LIU Gu-lan,et al. Application and development trend of SEC high-frequency chopper cascade governing technique[J]. Electric Switchgear, 2007, (6): 50-53.

[2] MA Yongguang, ZHANG Xiaodong, WANG Bingshu,et al. Dynamic model and simulation of cascade motor[J]. Electric Machines \& Control Application, 2007, 31(4): 11-14.

[3] ZHANG Junwei,WANG Bingshu, JIANG Ping. System modeling and double closed-loop control simulation in chopped wave cascade speed control system[J]. Control Engineering of China, 2011, 18(6): 977-981.

[4] JIANG Youhua, NING Yu, WU Guoxiang. Study on digital dual closed-loop chopper inner-feed cascade variable speed drive system based on state-space averaging model and its application[J]. Control Theory \& Applications, 2007, 24(1): 109-112. 